\title{
Modulation and elimination of yeast prions by protein chaperones and co-chaperones
}

\author{
Michael Reidy and Daniel C. Masison*
}

Laboratory of Biochemistry and Genetics; National Institute of Diabetes and Digestive and Kidney Diseases; National Institutes of Health; Bethesda, MD USA

Key words: prion, amyloid, chaperone, yeast, Hsp70, Hsp40, Hsp104

The yeast system has provided considerable insight into the biology of amyloid and prions. Here we focus on how alterations in abundance or function of protein chaperones and co-chaperones affect propagation of yeast prions. In spite of a considerable amount of information, a clear understanding of the molecular mechanisms underlying these effects remains wanting.

\section{Background}

Reed Wickner launched the yeast prion field in 1994 when he showed the non-Mendelian genetic element [URE3] propagated as an infectious form of the Ure2 protein. ${ }^{1}$ At the same time he proposed that another genetic element, $\left[\mathrm{PSI}^{+}\right]$, propagated as a prion of the Sup 35 protein. His discovery of the genetic identities of [URE3] and $\left[\mathrm{PSI}^{+}\right]$provided a simple and satisfying explanation for a large body of puzzling data regarding these elements. It also established yeast as a system for studying amyloid and the prion-like behavior of proteins.

The yeast system has since provided much insight into various aspects of prion biology. Having the advantages of facile and powerful genetics and molecular biology, the fungal prion field was first to confirm the prion hypothesis by demonstrating that proteins alone can act as infectious agents..$^{2-6}$ Meeting the genetic criteria for yeast prions defined by Wickner, several other yeast proteins also have been found to be capable of behaving as infectious proteins. Like their mammalian counterparts, the basis of the prion phenotype is the propensity of these proteins to misfold and form highly ordered fibrous aggregates called amyloid.

Since prions are improperly folded proteins, it is not surprising that alterations in abundance or function of protein chaperones and co-chaperones strongly influence yeast prion propagation. Many of these factors were identified on the basis of how their overexpression or depletion affects prion phenotypes. Molecular chaperones and co-chaperones overlap functionally, interact in complex ways, and their expression is feedback regulated. Therefore, it is difficult to know how altering abundance of any one of them influences cellular chaperone activity overall or whether any influence on prions can be attributed solely to the

*Correspondence to: Daniel C. Masison; Email: masisond@helix.nih.gov Submitted: 07/21/11; Accepted: 08/13/11

DOI: $10.4161 /$ pri.5.4.17749 effects of increasing or eliminating a specific activity of a particular chaperone. Moreover, the ways different prions respond to the same chaperone alterations are highly variable, even though all yeast prions so far characterized possess similar core structures. ${ }^{7-9}$ Given this complexity, it is easy to see why our understanding of the molecular mechanisms underlying most of these effects is limited.

What triggers appearance of yeast prions is uncertain, but once present they can propagate very stably under normal growth conditions. In order to persist in a growing population, yeast prions must grow, replicate and be transmitted to daughter cells (Fig. 1). Although it is widely accepted that yeast prion replication requires fragmentation of prion polymers by the action of the Hsp104 disaggregation machinery, to what extent chaperones might influence growth or transmission of prion polymers is much less clear.

\section{Hsp104}

Protein chaperones were first reported to be involved in yeast prion propagation when Yury Chernoff and colleagues showed that exogenously increasing Hsp104 abundance eliminates $\left[P S I^{+}\right] .{ }^{10}$ This study also showed that stable $\left[P S I^{+}\right]$propagation depends on normal uninduced levels of Hsp104. This requirement of Hsp104 is shared by the other amyloid-like yeast prions. However, none of these others are eliminated similarly by elevated Hsp104. ${ }^{11-14}$ Although this example of prion curing is unique to $\left[\mathrm{PSI}^{+}\right]$, it has been the basis of many findings regarding the involvement of several other factors in prion propagation.

Agreement in the field about the mechanism underlying the general requirement of Hsp104 for prion replication is that the disaggregation machinery acts directly on prion fibers to sever them, thereby generating new prion seeds from preexisting material. ${ }^{15-17}$ Hsp104 is a hexameric chaperone that resolubilizes proteins from aggregates by extruding them through an axial channel of the hexamer. ${ }^{18}$ This disaggregation reaction, which depends on Hsp70 and Hsp40, plays the major role in restoring viability after cells are exposed to severe stress (Fig. 2A). ${ }^{19-21}$ The efficiency of substrate threading by the Hsp104 machinery correlates directly with strength of $\left[\mathrm{PSI}^{+}\right]$propagation, indicating that this threading activity is also required for prion replication. ${ }^{22}$ Prion replication presumably occurs when Hsp104 machinery extracts monomers from a prion fiber, causing the fiber to become unstable and break into independently propagating prions 


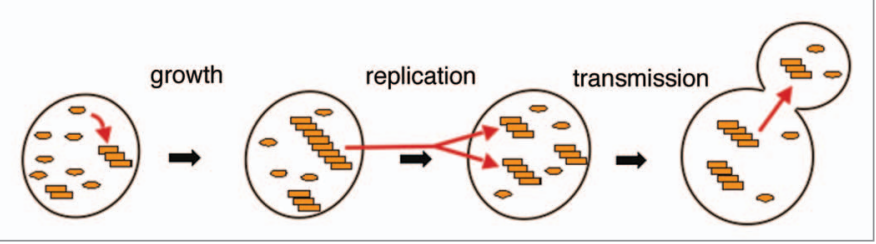

Figure 1. In order for prions to propagate stably in yeast they must grow, replicate and be transmitted efficiently to daughter cells (red arrows). Growth occurs when the soluble form of a prion protein (ovals) is converted into the prion conformation as it joins the end of a prion fiber (stack of rectangles). Replication occurs when prion fibers break into more numerous pieces that each can continue to propagate the prion conformation. Transmission of prions to daughter cells can occur by passive diffusion with cytoplasm.

(Fig. 2B). This replication process is crucial for stable prion propagation because if the number of prions per cell does not double in the time it takes cells to divide, then cells lacking the prion will arise in the population as eventually there will be more cells than prions.

The dependency of yeast prions on Hsp104 machinery for fragmentation implies that prion fibers are generally too sturdy to break often enough on their own to keep pace with cell division. $\left[\mathrm{PHI}^{+}\right]$, an exceptional hybrid prion that does not require Hsp104 to propagate stably, possibly confirms this notion. ${ }^{23}$ $\left[\mathrm{PHI}^{+}\right]$is formed of Sup35p containing a substitution in part of its prion-determining region. This modified Sup35p assembles into shorter than normal fibers in vitro, suggesting it might be fragile enough to break adequately without assistance from the Hsp104 machinery.

Although the mechanism of how the Hsp104 machinery acts in prion replication is well defined, our understanding of the molecular mechanisms underlying the many other ways that Hsp104 and other factors influence prion propagation is very limited. For example, mechanistic explanations of how overexpressed Hsp104 cures cells of $\left[\mathrm{PSI}^{+}\right]$and why the other prions are insensitive to this treatment remain unresolved. Data from in vitro and in vivo studies lead to different conclusions about how this curing occurs. Consistent with observations of how changes in Hsp104 abundance affect $\left[P S I^{+}\right]$, low amounts of Hsp104 promote nucleation and growth of Sup35p amyloid fibrils in vitro, but higher amounts block growth. ${ }^{24}$ These higher amounts also "exterminate" mature Sup35p amyloid fibrils, destroying their ability to induce $\left[\mathrm{PSI}^{+}\right]$when used to transfect yeast. In line with the specificity of $\left[\mathrm{PSI}^{+}\right]$curing, high amounts of Hsp104 had a similar destructive effect on Ure2p amyloid fibrils, but the resulting material retained residual infectivity to induce [URE3]. ${ }^{25}$ Invoking a simple mass action effect of increased disaggregation activity, the authors proposed that overexpressing Hsp104 cures cells of $\left[\mathrm{PSI}^{+}\right]$by completely dissolving Sup35p fibrils.

In vivo, however, SDS resistant Sup35p polymers appear to increase in size when Hsp104 is overexpressed. ${ }^{17}$ Additionally, elevating the substrate threading and protein resolubilizing functions of Hsp104 that are required for prion propagation are not enough to cause $\left[\mathrm{PSI}^{+}\right]$to be eliminated. ${ }^{22,26,27} \mathrm{Hsp} 104$ with a point mutation in its amino-terminal domain ( $\mathrm{Hsp} 104^{\mathrm{T} 160 \mathrm{M}}$ ) supports $\left[\mathrm{PSI}^{+}\right]$propagation like wild type Hsp104, indicating it acts normally on Sup35p prions as substrates. Hsp104 ${ }^{\mathrm{T} 160 \mathrm{M}}$ also functions normally in thermotolerance and reactivation of thermally denatured luciferase in vivo. ${ }^{22}$ Overexpressing Hsp104 ${ }^{\mathrm{T} 160 \mathrm{M}}$, however, does not affect $\left[P S I^{+}\right]$, implying that something in addition to these Hsp104 functions are required to cure cells of $\left[\mathrm{PSI}^{+}\right]$.

Recent findings support this interpretation. Recessive mutations in the Hsp70 co-chaperone Sislp that block the curing indicate that a specific Sislp activity is needed to cure cells of $\left[\mathrm{PSI}^{+}\right],{ }^{28}$ and factors that are not known to play a role in Hsp104 mediated thermotolerance or resolubilization of denatured proteins are also crucial for the curing. Reducing ubiquitin (Ub) levels reduces the curing, while increasing Ub enhances it. ${ }^{29,30}$ Sup35p is not ubiquitylated under either of these conditions, suggesting that Ub-mediated regulation or turnover of an unknown component is important for the curing process. The Hsp90 chaperone system, which serves as a post-translational regulator of many cellular processes, is also important for the curing mechanism. ${ }^{30,31}$ Increasing Hsp90 abundance does not affect curing by overexpressed Hsp104. ${ }^{32}$ However, although neither deleting Hsp70/ Hsp90 coordinating co-chaperone Stilp nor chemical inhibition of Hsp90 affect $\left[\mathrm{PSI}^{+}\right]$propagation, either treatment strongly inhibits the curing. ${ }^{30,31}$ Evidence suggests cell division is also required for the curing, ${ }^{30}$ which argues against curing by complete disaggregation since even a non-dividing cell overexpressing Hsp104 would be able to eliminate the prion if Hsp104 could simply dissolve it. Together these findings are difficult to reconcile with the conclusion that $\left[\mathrm{PSI}^{+}\right]$curing by overexpression of Hsp104 occurs simply by complete resolubilization of prion templates by Hsp104.

\section{Hsp70 and its Co-Chaperones}

Hsp70 and its obligate co-chaperone Hsp40 cooperate to bind exposed hydrophobic surfaces on partially folded proteins (Fig. 2A). This activity helps proteins adopt and maintain native conformation, provides a primary response to stress by preventing aggregation of misfolded proteins, and plays an essential role in many cellular processes such as translation and membrane transport. ${ }^{33}$ Many co-chaperones other than Hsp40 also regulate Hsp70 activity. These co-chaperones might influence activity of the Hsp104/Hsp70/Hsp40 machinery through their effects on Hsp70.

An influence of Hsp70 on yeast prions was first identified in a candidate gene approach when overexpression of the Hsp70 Ssalp was shown to inhibit Hsp104 curing of $\left[\mathrm{PSI}^{+}\right] .{ }^{32}$ Since balancing abundance of components of the same machinery might be expected to improve machinery function, this finding might not have been anticipated if curing was thought to be due to increased disaggregation. Increasing expression of Ssalp also enhances aggregation of Sup35p in $\left[\mathrm{PSI}^{+}\right]$cells, ${ }^{34}$ which again is counterintuitive given the important role of Hsp70 in preventing protein aggregation. Possible explanations for the inhibition of Hsp104 curing are that increasing Ssalp abundance promotes prion aggregation or protects prion fibers by binding them and 
restricting access to Hsp104. ${ }^{32,34}$ In support of the latter notion, Hsp70 and Hsp40 were found to be associated with Sup35p from $\left[\mathrm{PSI}^{+}\right]$cells, but not from $\left[p s i^{-}\right]$cells. ${ }^{35}$ This same interpretation explained the observations that although strength of $\left[\mathrm{PSI}^{+}\right]$phenotype is weakened by growth at elevated temperature, which induces expression of all three chaperones, normal $\left[\mathrm{PSI}^{+}\right]$prions are not cured efficiently by such treatment. Weak "strains" of $\left[\mathrm{PSI}^{+}\right]$, however, can be destabilized by a rapid shift to elevated temperature. ${ }^{36}$ Although this instability is presumed to be caused by transient imbalance of $\mathrm{Hsp} 104$ and $\mathrm{Hsp} 70$ abundance due to different rates of induction, the mechanisms of how variations of Hsp70 abundance influence curing of $\left[\mathrm{PSI}^{+}\right]$by elevated Hsp104 remain speculative.

A mutant Ssa1p, designated Ssa1-21p, destabilizes $\left[P S I^{+}\right]$in a manner very similar to that of elevated Hsp104. ${ }^{37}$ Several Hsp70 co-chaperones were identified as being important for Ssa1-21p to impair $\left[\mathrm{PSI}^{+}\right]$propagation. ${ }^{28,38}$ All of these are also important for Hsp104 curing of $\left[\mathrm{PSI}^{+}\right]$, implying that Ssa1-21p impairs $\left[P S I^{+}\right]$ by a mechanism similar to that of overexpressed Hsp104. ${ }^{28,30,31}$ Although the basis of this impairment has yet to be worked out, these findings suggest that Hsp70 and its co-chaperones contribute importantly to the mechanism of curing by overexpressed Hsp104.

Yeast have six canonical cytoplasmic Hsp70s; four of the essential Ssa subfamily and two of the ribosome-associated Ssb subfamily. Ssalp and Ssb1p are 64\% identical and overlap functionally, ${ }^{39,40}$ and both subfamilies function by the same generic ATP and co-chaperone regulated cycle of binding and releasing hydrophobic surfaces on protein substrates. When overexpressed, all four Ssa Hsp70s similarly inhibit $\left[P S I^{+}\right]$curing by $\mathrm{Hsp} 104$ and promote de novo formation of $\left[\mathrm{PSI}^{+}\right] .{ }^{34}$ Overexpressing Ssb, however, enhances prion curing by overexpressed Hsp104 and deleting Ssb increases de novo formation of $\left[\mathrm{PSI}^{+}\right] .{ }^{41}$ These findings suggest that despite their high homology, the Ssa and Ssb Hsp70s interact with Hsp104 or prions in different ways that determine whether Hsp104 influences $\left[\mathrm{PSI}^{+}\right]$propagation in a positive or negative manner.

Amino acid identity across the Ssa subfamily is greater than $80 \%$, so the common $\left[\mathrm{PSI}^{+}\right]$-promoting effect they have when they are overexpressed is not unexpected. However, when individual Ssa Hsp70s are expressed at physiological levels as the only Ssa Hsp70 in the cell, each of the four Ssa proteins affects the strength and stability of $\left[\mathrm{PSI}^{+}\right]$differently, ${ }^{42}$ showing that one and the same prion can be affected differently by highly homologous Hsp70s. In contrast to the effects on [PSI+], [URE3] propagation is impaired by overexpressing Ssalp. ${ }^{43}$ Yet, overexpressing the $98 \%$ identical Ssa2p does not affect [URE3]. ${ }^{43}$ Cells expressing individual Ssa proteins also display different [URE3] phenotypes, and again, the way three of the four Ssa Hsp70s affect [URE3] is opposite to way they affect $\left[\mathrm{PSI}^{+}\right] .{ }^{42}$ Thus, despite possessing the same reaction cycle and interacting similarly with substrates, nearly identical Hsp70s influence the same prion in different ways, and the ways different prions respond to individual Hsp70s is different.

These findings show not only that overexpressing individual Hsp70s can have very different effects on prions than expressing

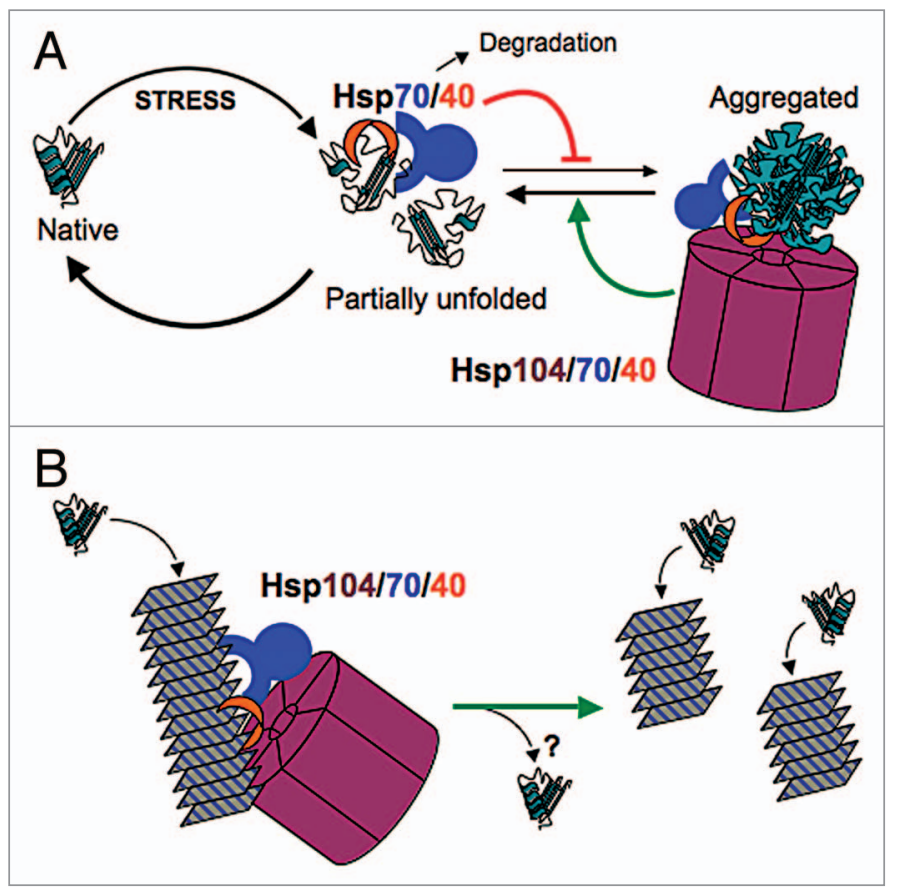

Figure 2. Models for the action of protein chaperones in stress protection (A) and yeast prion propagation (B). (A) Hsp70 (blue) and Hsp40 (red) prevent aggregation of proteins by binding and masking exposed hydrophobic surfaces on partially unfolded proteins. Release of the sub strate, catalyzed by nucleotide exchange factors (not shown), provides an opportunity for the protein to regain its native conformation. Hsp70 can also promote degradation of misfolded proteins. Hsp104 (purple), assisted by $\mathrm{Hsp} 70$ and $\mathrm{Hsp} 40$, resolubilizes proteins from aggregates by extruding individual polypeptides through its axial pore. The combined action of $\mathrm{Hsp} 70, \mathrm{Hsp} 40$ and $\mathrm{Hsp} 104$ drives the reaction toward the native state. (B) A similar reaction of the Hsp104/70/40 machine as in (A) but acting on a prion polymer, which is shown as stacked rectangles representing amyloid. Extraction of a monomer from within the polymer by the Hsp104/70/40 system destabilizes the fiber, allowing it to break into pieces, each of which continues to propagate the amyloid structure. The fate of the extracted monomer is uncertain (question mark). In order for the prion to be transmitted efficiently to daughter cells, new fibers must be created continually by the action of this chaperone machinery.

them at physiological levels, but also that these highly homologous Hsp70s each possess unique activities that distinctly influence some aspect of prion growth, replication or transmission. The bases for these differences is not yet known, but given the wide range of roles Hsp70s play in the cell they might be indirect. Regardless of the explanation, the mechanisms likely involve differences in ways the Hsp70s interact with and are regulated by co-chaperones.

Several co-chaperones that regulate Hsp70 influence prions in various ways. ${ }^{12,38,44-46}$ S. cerevisiae has 13 cytosolic Hsp40s, which promote $\mathrm{Hsp} 70$ binding to substrates. ${ }^{47}$ Overexpressing the Hsp40 Ydj1p cures cells of [URE3], but not $\left[\mathrm{PSI}^{+}\right] .^{12}$ Mutagenesis of Ydj1p showed its interaction with Hsp70 is both necessary and sufficient for the curing. ${ }^{48}$ Efficiency of this curing also depends on interaction of Ydj1p with specific Ssa Hsp70s. ${ }^{49}$ Depleting the essential Hsp40 Sis1p causes rapid and efficient loss of [URE3] and $\left[\mathrm{PIN}^{+}\right] /[R N Q 1]$ prions, but delayed and 
gradual loss of $\left[P S I^{+}\right] .^{50}$ Certain Hsp40 functions of Sislp are required for propagation of $\left[\mathrm{PIN}^{+}\right]$and are critical for curing of $\left[\mathrm{PSI}^{+}\right]$by Hsp104 overexpression. ${ }^{28,44}$ Yet, these and other Sislp activities are dispensable for normal propagation of $\left[\mathrm{PSI}^{+}\right]$, suggesting other Hsp40s can substitute for Sislp to support $\left[P S I^{+}\right] .{ }^{28}$ The proposed role of Sislp in prion replication is to recruit Hsp70 and Hsp104 to prion polymers. ${ }^{51}$ The finding that Sislp lacking its substrate binding region supports normal $\left[\mathrm{PSI}^{+}\right]$propagation is inconsistent with this proposal. ${ }^{28} \mathrm{Fes} 1 \mathrm{p}$ and Sselp are nucleotide exchange factors that help Hsp70 release substrates. ${ }^{52,53}$ Overexpressing Sselp destabilizes [URE3] and inhibits [PSI+] curing by Hsp104, and deleting either Feslp or Sselp can destabilize both [URE] and $\left[P S I^{+}\right] .38,45,46,54$ Whenever tested, the ability of these co-chaperones to regulate $\mathrm{Hsp} 70$ was found to be necessary for them to exert their effects on prions. These findings show that alterations in proteins that regulate Hsp70 activity influence different prions in different ways, that effects of co-chaperones on prions are mediated through general and specific interactions with Hsp70, and again that chaperone activities important for prion propagation and elimination can be distinct.

Despite all this information, the complexity of the ways chaperones and co-chaperones interact and regulate each other's activities and expression has made it difficult to say with confidence how their effects on prions are mediated at a molecular level. Adding to the difficulty are the differences in the ways various prions and even "strains" of the same prion respond to identical chaperone alterations. What mechanism would satisfyingly explain how structurally related prions can respond oppositely to the same chaperone alteration? On the whole, very few chaperone alterations produce general and predictable effects on prions. The only trait that native yeast amyloid-based prions display to a similar degree is their dependency on Hsp104 for replication (although the nuclear-localized prion of Sfplp, designated [ISP+ ${ }^{+}$, has the unusual property of being eliminated by the Hsp104inactivating compound guanidine- $\mathrm{HCl}$, but not by deletion of the Hsp104 gene). ${ }^{55}$ Since Hsp70 and Hsp40 assist Hsp104, it is possible that effects they have on the different prions are mediated through an effect on Hsp104 function. If so, then the various ways $\mathrm{Hsp} 70$ and its co-chaperones affect prions could point to differences in the ways the different prions rely on this machinery, perhaps determined by variations in amino acid sequence or particular configuration of prion polymers. Alternatively, Hsp70, Hsp40 and other co-chaperones might influence cellular processes that yeast prions rely on for stable propagation. Disrupting actin dynamics, for example, strongly influences $\left[\mathrm{PSI}^{+}\right]$phenotypes. ${ }^{56,57}$ An important challenge is to define more clearly the cellular and molecular events that produce the various effects that chaperones have on prion propagation. Meeting the challenge will undoubtedly require determining if $\mathrm{Hsp} 70$ and its cochaperones affect prions directly or indirectly, and to what extent chaperones specifically affect the processes of prion growth, replication and transmission.

\section{Acknowledgments}

We thank NIH colleagues for critical reading of the manuscript. This work was supported by the Intramural Research Program of the National Institutes of Health, National Institute of Diabetes and Digestive and Kidney Diseases.

\section{References}

1. Wickner RB. Evidence for a prion analog in S. cerevisiae: the [URE3] non-Mendelian genetic element as an altered URE2 protein. Science 1994; 264:566-9.

2. Maddelein ML, Dos Reis S, Duvezin-Caubet S, Coulary-Salin B, Saupe SJ. Amyloid aggregates of the HET-s prion protein are infectious. Proc Natl Acad Sci USA 2002; 99:7402-7.

3. King CY, Diaz-Avalos R. Protein-only transmission of three yeast prion strains. Nature 2004; 428:319-23.

4. Tanaka M, Chien P, Naber N, Cooke R, Weissman JS. Conformational variations in an infectious protein determine prion strain differences. Nature 2004; 428:323-8.

5. Brachmann A, Baxa U, Wickner RB. Prion generation in vitro: amyloid of Ure2p is infectious. EMBO J 2005; 24:3082-92.

6. Patel BK, Liebman SW. "Prion-proof" for [PIN ${ }^{+}$; infection with in vitro-made amyloid aggregates of Rnqlp-(132-405) induces [PIN ${ }^{+}$. J Mol Biol 2007; 365:773-82.

7. Baxa U, Wickner RB, Steven AC, Anderson DE, Marekov LN, Yau WM, Tycko R. Characterization of beta-sheet structure in Ure2p1-89 yeast prion fibrils by solid-state nuclear magnetic resonance. Biochemistry 2007; 46:13149-62.

8. Shewmaker F, Wickner RB, Tycko R. Amyloid of the prion domain of Sup35p has an in-register parallel beta-sheet structure. Proc Natl Acad Sci USA 2006; 103:19754-9.

9. Wickner RB, Dyda F, Tycko R. Amyloid of Rnq1p, the basis of the $\left[\mathrm{PIN}^{+}\right]$prion, has a parallel in-register beta-sheet structure. Proc Natl Acad Sci USA 2008; 105:2403-8.
10. Chernoff YO, Lindquist SL, Ono B, Inge-Vechtomov SG, Liebman SW. Role of the chaperone protein Hsp104 in propagation of the yeast prion-like factor [psi ${ }^{+}$. Science 1995; 268:880-4.

11. Derkatch IL, Bradley ME, Zhou P, Chernoff YO, Liebman SW. Genetic and environmental factors affecting the de novo appearance of the $\left[\mathrm{PSI}^{+}\right]$prion in Saccharomyces cerevisiae. Genetics 1997; 147:507-19.

12. Moriyama H, Edskes HK, Wickner RB. [URE3] prion propagation in Saccharomyces cerevisiae: requirement for chaperone Hsp104 and curing by overexpressed chaperone Ydj1p. Mol Cell Biol 2000; 20:8916-22.

13. Du Z, Park KW, Yu H, Fan Q, Li L. Newly identified prion linked to the chromatin-remodeling factor Swil in Saccharomyces cerevisiae. Nat Genet 2008; 40:460-5.

14. Patel BK, Gavin-Smyth J, Liebman SW. The yeast global transcriptional co-repressor protein $\mathrm{Cyc} 8 \mathrm{can}$ propagate as a prion. Nat Cell Biol 2009; 11:344-9.

15. Paushkin SV, Kushnirov VV, Smirnov VN, TerAvanesyan MD. Propagation of the yeast prion-like [psi $\left.{ }^{+}\right]$determinant is mediated by oligomerization of the SUP35-encoded polypeptide chain release factor. EMBO J 1996; 15:3127-34.

16. Ness F, Ferreira P, Cox BS, Tuite MF. Guanidine hydrochloride inhibits the generation of prion "seeds" but not prion protein aggregation in yeast. Mol Cell Biol 2002; 22:5593-605.

17. Kryndushkin DS, Alexandrov IM, Ter-Avanesyan MD Kushnirov VV. Yeast $\left[\mathrm{PSI}^{+}\right]$prion aggregates are formed by small Sup35 polymers fragmented by Hsp104. J Biol Chem 2003; 278:49636-43.

18. Glover JR, Lum R. Remodeling of protein aggregates by Hsp104. Protein Pept. Lett 2009; 16:587-97.

19. Parsell DA, Kowal AS, Singer MA, Lindquist S. Protein disaggregation mediated by heat-shock protein Hsp104. Nature 1994; 372:475-8.
20. Glover JR, Lindquist S. Hsp104, Hsp70 and Hsp40: novel chaperone system that rescues previously aggregated proteins. Cell 1998; 94:73-82.

21. Lum R, Tkach JM, Vierling E, Glover JR. Evidence for an unfolding/threading mechanism for protein disaggregation by Saccharomyces cerevisiae Hsp104. J Biol Chem 2004; 279:29139-46.

22. Hung GC, Masison DC. N-terminal domain of yeas Hsp104 chaperone is dispensable for thermotolerance and prion propagation but necessary for curing prions by Hsp104 overexpression. Genetics 2006; 173:611-20.

23. Crist CG, Nakayashiki T, Kurahashi H, Nakamura Y. $\left[\mathrm{PHI}^{+}\right]$, a novel Sup35-prion variant propagated with non-Gln/Asn oligopeptide repeats in the absence of the chaperone protein Hsp104. Genes Cells 2003; 8:603-18.

24. Shorter J, Lindquist S. Hsp104 catalyzes formation and elimination of self-replicating Sup35 prion conformers. Science 2004; 304:1793-7.

25. Shorter J, Lindquist S. Destruction or potentiation of different prions catalyzed by similar Hsp104 remodeling activities. Mol Cell 2006; 23:425-38.

26. Jung G, Jones G, Masison DC. Amino acid residue 184 of yeast Hsp104 chaperone is critical for prion-curing by guanidine, prion propagation and thermotolerance. Proc Natl Acad Sci USA 2002; 99:9936-41.

27. Tessarz P, Mogk A, Bukau B. Substrate threading through the central pore of the Hsp104 chaperone as a common mechanism for protein disaggregation and prion propagation. Mol Microbiol 2008; 68:87-97.

28. Kirkland PA, Reidy M, Masison DC. Functions of yeast Hsp40 chaperone Sis1p dispensable for prion propagation but important for prion curing and protection from prion toxicity. Genetics 2011; 188:565-77.

29. Allen KD, Chernova TA, Tennant EP, Wilkinson KD, Chernoff YO. Effects of ubiquitin system alterations on the formation and loss of a yeast prion. J Biol Chem 2007; 282:3004-13 
30. Reidy M, Masison DC. Sti1 regulation of Hsp70 and Hsp90 is critical for curing of Saccharomyces cerevisiae $\left[\mathrm{PSI}^{+}\right]$prions by Hsp104. Mol Cell Biol 2010; 30:3542-52.

31. Moosavi B, Wongwigkarn J, Tuite MF. Hsp70/Hsp90 co-chaperones are required for efficient Hsp104mediated elimination of the yeast $\left[\mathrm{PSI}^{+}\right]$prion but not for prion propagation. Yeast 2010; 27:167-79.

32. Newnam GP, Wegrzyn RD, Lindquist SL, Chernoff YO. Antagonistic interactions between yeast chaperones Hsp104 and Hsp70 in prion curing. Mol Cell Biol 1999; 19:1325-33.

33. Meimaridou E, Gooljar SB, Chapple JP. From hatching to dispatching: the multiple cellular roles of the Hsp70 molecular chaperone machinery. J Mol Endocrinol 2009; 42:1-9.

34. Allen KD, Wegrzyn RD, Chernova TA, Muller S, Newnam GP, Winslett PA, et al. Hsp70 chaperones as modulators of prion life cycle: novel effects of Ssa and Ssb on the Saccharomyces cerevisiae prion $\left[\mathrm{PSI}^{+}\right]$. Genetics 2005; 169:1227-42.

35. Bagriantsev SN, Gracheva EO, Richmond JE, Liebman SW. Variant-specific $\left[\mathrm{PSI}^{+}\right]$infection is transmitted by Sup35 polymers within $\left[\mathrm{PSI}^{+}\right]$aggregates with heterogeneous protein composition. Mol Biol Cell 2008; 19:2433-43.

36. Newnam GP, Birchmore JL, Chernoff YO Destabilization and recovery of a yeast prion after mild heat shock. J Mol Biol 2011; 408:432-48.

37. Jung G, Jones G, Wegrzyn RD, Masison DC. A role for cytosolic $\mathrm{Hsp} 70$ in yeast $\left[\mathrm{PSI}^{+}\right]$prion propagation and $\left[\mathrm{PSI}^{+}\right]$as a cellular stress. Genetics 2000; 156:559-70.

38. Jones G, Song Y, Chung S, Masison DC. Propagation of Saccharomyces cerevisiae $\left[\mathrm{PSI}^{+}\right]$prion is impaired by factors that regulate Hsp70 substrate binding. Mol Cell Biol 2004; 24:3928-37.

39. Boorstein WR, Ziegelhoffer T, Craig EA. Molecular evolution of the HSP70 multigene family. J Mol Evol 1994; 38:1-17.
40. James P, Pfund C, Craig EA. Functional specificity among Hsp70 molecular chaperones. Science 1997; 275:387-9.

41. Chernoff YO, Newnam GP, Kumar J, Allen K, Zink $\mathrm{AD}$. Evidence for a protein mutator in yeast: role of the Hsp70-related chaperone ssb in formation, stability and toxicity of the [PSI] prion. Mol Cell Biol 1999; 19:8103-12.

42. Sharma D, Masison DC. Functionally redundant isoforms of a yeast $\mathrm{Hsp} 70$ chaperone subfamily have different antiprion effects. Genetics 2008; 179:1301-11.

43. Schwimmer C, Masison DC. Antagonistic interaction between yeast $\left[\mathrm{PSI}^{+}\right]$and [URE3] prions and curing of [URE3] by Hsp70 protein chaperone Ssalp but not by Ssa2p. Mol Cell Biol 2002; 22:3590-8.

44. Sondheimer N, Lopez N, Craig EA, Lindquist $S$. The role of Sis1 in the maintenance of the $\left[\mathrm{RNQ}^{+}\right]$prion. EMBO J 2001; 20:2435-42.

45. Fan Q, Park KW, Du Z, Morano KA, Li L. The role of Sse1 in the de novo formation and variant determination of the $\left[\mathrm{PSI}^{+}\right]$prion. Genetics 2007; 177:1583-93.

46. Kryndushkin D, Wickner RB. Nucleotide exchange factors for Hsp70s are required for [URE3] prion propagation in Saccharomyces cerevisiae. Mol Biol Cell 2007; 18:2149-54.

47. Craig EA, Huang P, Aron R, Andrew A. The diverse roles of J-proteins, the obligate Hsp70 co-chaperone. Rev Physiol Biochem Pharmacol 2006; 156:1-21.

48. Sharma D, Stanley RF, Masison DC. Curing of yeast [URE3] prion by the Hsp40 cochaperone Ydj1p is mediated by Hsp70. Genetics 2009; 181:129-37.

49. Sharma D, Martineau CN, Le Dall MT, Reidy M, Masison DC, Kabani M. Function of SSA subfamily of Hsp70 within and across species varies widely in complementing Saccharomyces cerevisiae cell growth and prion propagation. PLoS One 2009; 4:6644.
50. Higurashi T, Hines JK, Sahi C, Aron R, Craig EA. Specificity of the J-protein Sis 1 in the propagation of 3 yeast prions. Proc Natl Acad Sci USA 2008; 105:16596-601.

51. Tipton KA, Verges KJ, Weissman JS. In vivo monitoring of the prion replication cycle reveals a critical role for Sis1 in delivering substrates to Hsp104. Mol Cell 2008; 32:584-91.

52. Kabani M, Beckerich JM, Brodsky JL. Nucleotide exchange factor for the yeast Hsp70 molecular chaperone Ssalp. Mol Cell Biol 2002; 22:4677-89.

53. Raviol H, Sadlish H, Rodriguez F, Mayer MP, Bukau B. Chaperone network in the yeast cytosol: Hsp110 is revealed as an Hsp70 nucleotide exchange factor. EMBO J 2006; 25:2510-8.

54. Sadlish H, Rampelt H, Shorter J, Wegrzyn RD, Andreasson C, Lindquist S, et al. Hsp110 chaperones regulate prion formation and propagation in $S$. cerevisiae by two discrete activities. PLoS One 2008; 3:1763.

55. Rogoza T, Goginashvili A, Rodionova S, Ivanov $M$, Viktorovskaya O, Rubel A, et al. Non-Mendelian determinant $\left[I S P^{+}\right]$in yeast is a nuclear-residing prion form of the global transcriptional regulator Sfp1. Proc Natl Acad Sci USA 2010; 107:10573-7.

56. Bailleul-Winslett PA, Newnam GP, Wegrzyn RD, Chernoff YO. An antiprion effect of the anticytoskeletal drug latrunculin A in yeast. Gene Expr 2000; 9:145-56.

57. Ganusova EE, Ozolins LN, Bhagat S, Newnam GP, Wegrzyn RD, Sherman MY, et al. Modulation of prion formation, aggregation and toxicity by the actin cytoskeleton in yeast. Mol Cell Biol 2006; 26:617-29. 